Irish Math. Soc. Bulletin

Number 69, Summer 2012, 9-9

ISSN 0791-5578

\title{
RANGES OF BIMODULE PROJECTIONS AND CONDITIONAL EXPECTATIONS
}

\author{
ROBERT PLUTA
}

This is an abstract of the $\mathrm{PhD}$ thesis Ranges of Bimodule Projections and Conditional Expectations written by R. Pluta under the supervision of Prof. Richard M. Timoney at the School of Mathematics, Trinity College Dublin and submitted in September 2011.

The algebraic theory of corner rings introduced by Lam [1] (as an abstraction of the properties of Peirce corners $e$ Re of a ring $R$ associated with an idempotent $e \in R$ ) is investigated in the context of $C^{*}$-algebras and operator algebras. The main result is as follows.

Theorem. Let $H$ be a Hilbert space with an orthonormal basis $\left(e_{i}\right)_{i \in I}$ (which may be countable or uncountable), and $\mathcal{B}(H)$ the algebra of bounded operators on $H$. Let $\mathcal{E}: \mathcal{B}(H) \rightarrow \mathcal{B}(H)$ be a linear map with range $S$ a subalgebra such that $\mathcal{E} \circ \mathcal{E}=\mathcal{E}, \mathcal{E}$ is an $S$-bimodule map, and $\mathcal{E}\left(x^{*}\right)=\mathcal{E}(x)^{*}$ for $x \in \mathcal{B}(H)$ ( $\mathcal{E}$ is called a Lam conditional expectation). Then, if $e_{i} \otimes e_{i}^{*} \in S$ for $i \in I$, there is an equivalence relation on I such that $\mathcal{E}(x)=\sum_{j \in J} p_{j} x p_{j}$ for $x \in \mathcal{B}(H)$, where $J$ is the set of equivalence classes, $p_{j}=\sum_{i \in j} e_{i} \otimes e_{i}^{*}$ for $j \in J$, and $e_{i} \otimes e_{i}^{*}$ is the operator that sends an element $h \in H$ to $\left\langle h, e_{i}\right\rangle e_{i} \in H$.

This is generalized to purely atomic von Neumann algebras.

\section{REFERENCES}

[1] T. Y. Lam: Corner ring theory: a generalization of Peirce decompositions. I. Algebras, rings and their representations, 153-182, World Sci. Publ., Hackensack, NJ, 2006.

School of Mathematics, Trinity College Dublin

E-mail address, R. Pluta: plutar@tcd.ie

2010 Mathematics Subject Classification. 46L05, 46L07.

Key words and phrases. $C^{*}$ algebra, injective, noncommutative conditional expectation.

Received on 14-5-2012.

I would like to thank Prof. Richard M. Timoney for the care he took in supervising my $\mathrm{PhD}$ thesis. 